\title{
Synthesis and Photochromism of Novel Phenylene-linked Photochromic Bispyrans
}

\author{
Weili Zhao and Erick M. Carreira* \\ Laboratorium für Organische Chemie \\ ETH-Hönggerberg HCI H335 \\ CH-8093 Zürich, Switzerland. \\ Carreira@org.chem.ethz.ch
}

\section{Supplementary Material}

\section{General Information:}

All reactions were performed using oven dried glassware. 1,2-dichloroethane and DMSO were dried over molecular sieves $(3 \AA$ ). THF were dried by passing through two $4 \times 36$ inch columns of anhydrous neutral A-2 alumina $(8 \times 14$ mesh; Macherey und Nagel; activated under a flow of $\mathrm{N}_{2}$ at $300{ }^{\circ} \mathrm{C}$ over night) to remove water. Benzene was dried with sodium and distilled. All chemicals were purchased from Aldrich and Fluka, and used as such. The solvents used for UV-vis, IR and fluorescence study are all spectroscopic grade. 4-Hydroxy-1-phenyl-naphthoic acid ethyl ester, ${ }^{1} 3,3$-Diphenyl-[3H]naphtho[2,1-b]pyran, ${ }^{2}$ and 2,2,6-triphenyl-5-ethoxycarbonyl-[2H]naphtho[1,2-b]pyran ${ }^{2}$ were prepared by the known procedure.

For chromatography, technical grade solvents were used, which were distilled prior to use. Chromatographic purification was performed as flash chromatography using Brunschwig silica 32-63, $60 \AA$, or MN-aluminiumoxide (neutral, deactivated with water to activity III) with $0.3-0.5$ bar pressure.

${ }^{1} \mathrm{H}$ NMR spectra were recorded on a VARIAN Mercury $300 \mathrm{MHz}$ spectrometer in chloroform-d, all signals are reported in ppm with the internal chloroform signal at 7.26 ppm. The data is being reported as $(\mathrm{s}=$ singlet, $\mathrm{d}=$ doublet, $\mathrm{t}=$ triplet, $\mathrm{q}=$ quartet, $\mathrm{m}=$ multiplet, coupling constant(s), integration). ${ }^{13} \mathrm{C}$ NMR spectra were recorded with ${ }^{1} \mathrm{H}$ decoupling on a VARIAN Mercury $75 \mathrm{MHz}$ spectrometer in chloroform-d, all signals are reported in ppm with the internal chloroform signal at $77.0 \mathrm{ppm}$ as standard.

Infrared spectra were recorded on a Perkin Elmer Spectrum RX-I FT-IR spectrophotometer as thin films or as solution in chloroform and are reported as $\mathrm{cm}^{-1}$. Mass spectrometric measurements were performed by the mass spectrometry service of the LOC at the ETHZ.

UV-vis spectra were recorded in Varian Cary $50 \mathrm{UV}$-vis spectrometer equipped with thermostated sell holder with a magnetic stirrer underneath. The temperature was 
controlled with RM6 LAUDA thermo-cryostat. The temperature inside the cuvette was measured with EBRO TFX 592 digital thermometer.

Photoreaction was generally carried out in quartz container irradiated with either $8 \mathrm{~W}$ or $40 \mathrm{~W}$ CAMAG UV lamp, or with $150 \mathrm{~W}$ medium pressure Hg lamp from Heraeus Labor UV-Reaktorsystem.

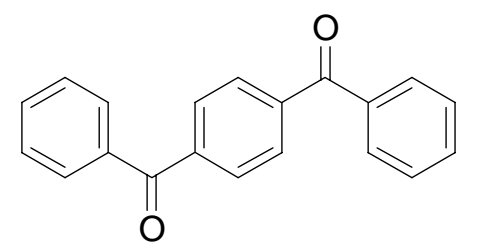

4a, 1,4-dibenzoyl-benzene

$\mathrm{AlCl}_{3}(2.7 \mathrm{~g}, 20.5 \mathrm{mmol})$ was added in small portions to a solution of terephthaloyl chloride $(2.0 \mathrm{~g}, 10 \mathrm{mmol})$ in dry benzene $(20 \mathrm{~mL})$ with stirring at room temperature. After stirring for $20 \mathrm{~h}$, the reaction mixture was poured on to crushed ice. Excess benzene was evaporated, the resulted solid was filtered, washed with water, then with $10 \%$ aqueous $\mathrm{NaOH}$, followed by water. The solid $(2.55 \mathrm{~g}, 89 \%)$ was used for the next step without further purification.

${ }^{1} \mathrm{H}$ NMR (300 MHz, $\left.\mathrm{CDCl}_{3}\right): \delta 7.89$ (s, 4H), 7.85-7.81 (m, 4H), 7.67-7.60 (m, 2H), 7.56$7.48(\mathrm{~m}, 5 \mathrm{H})$.

${ }^{13} \mathrm{C}$ NMR $\left(75 \mathrm{MHz}, \mathrm{CDCl}_{3}\right): \delta 195.7,140.5,136.8,132.8,130.0,129.6,128.3$.

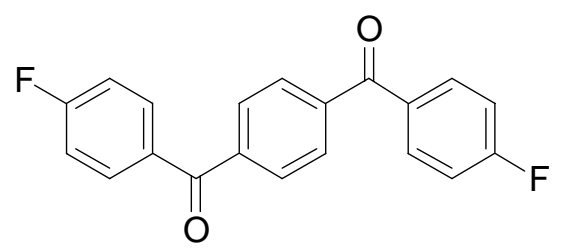

4b, 1,4-di( $p$-fluorobenzoyl)-benzene

$\mathrm{AlCl}_{3}(7.0 \mathrm{~g}, 52 \mathrm{mmol})$ was added in small portions to a solution of terephthaloyl chloride $(5.0 \mathrm{~g}, 25 \mathrm{mmol})$ in fluorobenzene $(28.0 \mathrm{~g}, 29 \mathrm{mmol})$ with stirring at room temperature. After stirring over $4 \mathrm{~h}$, the reaction mixture was poured onto crushed ice. Excess benzene was evaporated, the resulted solid was filtered, washed with water, then with $10 \%$ aqueous $\mathrm{NaOH}$, followed by water. White solid (7.72 g, 96\%) obtained was used for the next step without further purification.

IR $\left(\mathrm{CHCl}_{3}\right)$ : 3030, 2927, 1662, 1600, 1508, 1498, 1408, 1303, 1276, 1242, 1155, 926, 845.

${ }^{1} \mathrm{H}$ NMR (300 MHz, acetone): $\delta$ 7.95-7.91 (m, 4H), 7.92 (s, 4H), 7.39-7.30 (m, 4H).

${ }^{13} \mathrm{C}$ NMR $\left(75 \mathrm{MHz}, \mathrm{CDCl}_{3}\right): \delta 194.4,165.7\left({ }^{1} \mathrm{~J}=255.1 \mathrm{~Hz}\right), 140.6,133.1\left({ }^{4} \mathrm{~J}=3.1 \mathrm{~Hz}\right)$, $132.8\left({ }^{3} \mathrm{~J}=9.2 \mathrm{~Hz}\right), 129.6,115.7\left({ }^{2} \mathrm{~J}=22.0 \mathrm{~Hz}\right)$.<smiles>COc1ccc(C(=O)c2ccc(C(=O)c3ccc(OC)cc3)cc2)cc1</smiles>

4c, 1,4-di( $p$-methoxybenzoyl)-benzene 
$\mathrm{AlCl}_{3}(2.9 \mathrm{~g}, 22 \mathrm{mmol})$ was added in small portions to a solution of terephthaloyl chloride $(2.0 \mathrm{~g}, 10 \mathrm{mmol})$ and anisole $(5.4 \mathrm{~g}, 5 \mathrm{mmol})$ in dichloromethane $(7 \mathrm{~mL})$ with stirring at room temperature. After stirring over $20 \mathrm{~h}$, the reaction mixture was poured on to crushed ice. Excess benzene was evaporated, the resulted solid was filtered, washed with water, then with $10 \%$ aqueous $\mathrm{NaOH}$, followed by water. White solid $(2.98 \mathrm{~g}, 86 \%)$ obtained was used for the next step without further purification.

IR $\left(\mathrm{CHCl}_{3}\right): 3019,2937,2842,1650,1601,1511,1464,1316,1281,1260,1176,1156$, $1032,927$.

${ }^{1} \mathrm{H}$ NMR $\left(300 \mathrm{MHz}, \mathrm{CDCl}_{3}\right): \delta$ 7.90-7.83 (m, 4H), $7.83(\mathrm{~s}, 4 \mathrm{H}), 7.02-6.95(\mathrm{~m}, 4 \mathrm{H}), 3.91$ $(\mathrm{s}, 6 \mathrm{H})$.

${ }^{13} \mathrm{C} \mathrm{NMR}\left(75 \mathrm{MHz}, \mathrm{CDCl}_{3}\right): \delta 194.6,163.4,140.9,132.5,120.6,55.6$.<smiles>O=C(c1ccccc1)c1cccc(C(=O)c2ccccc2)c1</smiles>

4'a, 1,3-dibenzoyl-benzene

The procedure of $\mathbf{4 a}$ was followed except that isoterephthaloyl chloride was used instead of terephthaloyl chloride, and white solid $(2.83 \mathrm{~g}, 99 \%)$ obtained was used for the next step without further purification.

IR $\left(\mathrm{CHCl}_{3}\right):$ 3066, 3030, 3014, 2927, 1661, 1599, 1579, 1448, 1322, 1259, 1171, 1124, $985,784,713$.

${ }^{1} \mathrm{H}$ NMR $\left(300 \mathrm{MHz}, \mathrm{CDCl}_{3}\right): \delta 8.21-8.17(\mathrm{~m}, 1 \mathrm{H}), 8.04(\mathrm{~d}, \mathrm{~J}=1.8 \mathrm{~Hz}, 1 \mathrm{H}), 8.02(\mathrm{~d}, \mathrm{~J}=$ $1.8 \mathrm{~Hz}, 1 \mathrm{H}), 7.86-7.79(\mathrm{~m}, 4 \mathrm{H}), 7.67-7.57(\mathrm{~m}, 3 \mathrm{H}), 7.55-7.45(\mathrm{~m}, 4 \mathrm{H})$.

${ }^{13} \mathrm{C}$ NMR $\left(75 \mathrm{MHz}, \mathrm{CDCl}_{3}\right): \delta 195.5,137.6,136.8,133.3,132.7,131.1,129.9,128.3$.<smiles>O=C(c1ccc(F)cc1)c1cccc(C(=O)c2ccc(F)cc2)c1</smiles>

4'b, 1,3-di( $p$-fluorobenzoyl)benzene

The procedure of $\mathbf{4 b}$ was followed except that isoterephthaloyl chloride was used instead of terephthaloyl chloride. White solid $(7.92 \mathrm{~g}, 98 \%)$ obtained was used for the next step without further purification.

IR $\left(\mathrm{CHCl}_{3}\right): 3030,3018,2927,1662,1600,1506,1425,1410,1314,1298,1256,1242$, $1156,988,852,735,705$.

${ }^{1} \mathrm{H}$ NMR (300 MHz, acetone): $\delta$ 8.12-8.08 (m, 1H), 8.05 (dd, J = 7.8, $\left.1.6 \mathrm{~Hz}, 2 \mathrm{H}\right), 7.98-$ 7.89 (m, 4H), 7.77 (ddd, J = 8.1, 7.8, 1.6 Hz, 1H), 7.38-7.28 (m, 4H).

${ }^{13} \mathrm{C}$ NMR $\left(75 \mathrm{MHz}, \mathrm{CDCl}_{3}\right): \delta 194.0,165.4\left({ }^{1} \mathrm{~J}=255.1 \mathrm{~Hz}\right), 137.6,133.2,133.0\left({ }^{4} \mathrm{~J}=3.1\right.$ $\mathrm{Hz}), 132.6\left({ }^{3} \mathrm{~J}=9.2 \mathrm{~Hz}\right), 130.7,128.5,115.6\left({ }^{1} \mathrm{~J}=22.0 \mathrm{~Hz}\right)$.

\section{General Procedure for the preparation of bispropargyl alcohols}

Bisketone $(5.65 \mathrm{mmol})$ was added in one portion to a suspension of sodium acetylide $(22.67 \mathrm{mmol})$ in dry THF $(20 \mathrm{~mL})$ with stirring while acetylene gas was bubbled. DMSO $(5 \mathrm{~mL})$ was added. The mixture was stirred at room temperature for $3 \mathrm{~h}$ while keeping bubbling of acetylene. The mixture was allowed to stir overnight, poured onto crush ice 
and extracted with dichloromethane. After removal of solvent, the residue was purified by chromatography and/or be recrystalization from dichloromethane/hexane.

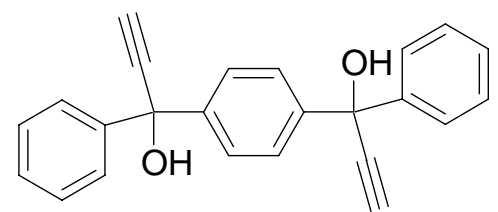

5a, 1,4-di(1-hydroxy-1-phenyl-prop-2-ynyl)benzene

The general procedure was followed using 1,4-dibenzoyl benzene as starting material, beige solid was obtained in yield of $72.6 \%$.

mp $187-189.5^{\circ} \mathrm{C}$.

IR $\left(\mathrm{CHCl}_{3}\right): 3587,3305,3064,3013,2926,1600,1490,1449,1408,1326,1162,1045$, 986, 892, 832 .

${ }^{1} \mathrm{H}$ NMR $\left(300 \mathrm{MHz}, \mathrm{CDCl}_{3}\right): \delta$ 7.63-7.55 (m, 5H), 7.37-7.23 (m, 2H), $2.87(\mathrm{~s}, 2 \mathrm{H}), 2.79$ $(\mathrm{s}, 2 \mathrm{H})$.

${ }^{13} \mathrm{C}$ NMR $\left(75 \mathrm{MHz}, \mathrm{CDCl}_{3}\right): \delta 144.0,143.8,128.2,127.8,125.9,125.8,86.2,75.6,74.1$.

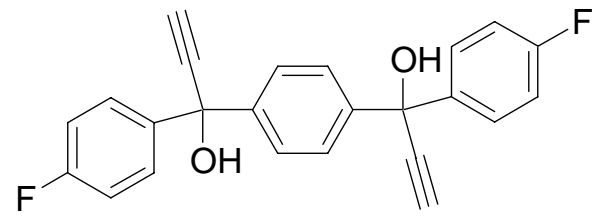

5b, 1,4-di[1-hydroxy-1-(p-fluorophenyl)-prop-2-ynyl]benzene

The general procedure was followed using 1,4-di(p-fluorobenzoyl) benzene as starting material, yellow-orange gel was obtained in yield of $61 \%$.

IR $\left(\mathrm{CHCl}_{3}\right)$ : 3587, 3305, 3019, 1603, 1508, 1411, 1325, 1228, 1160, 1052, 988, 900, 837.

${ }^{1} \mathrm{H}$ NMR $\left(300 \mathrm{MHz}, \mathrm{CDCl}_{3}\right): \delta$ 7.60-7.50 (m, 8H), 7.04-6.94 (m, 4H), $3.09(\mathrm{~s}, 2 \mathrm{H}), 2.87$ (s, 2H),

${ }^{13} \mathrm{C}$ NMR $\left(75 \mathrm{MHz}, \mathrm{CDCl}_{3}\right): \delta 162.1\left({ }^{1} \mathrm{~J}=246.6 \mathrm{~Hz}\right), 160.4,143.8,139.8\left({ }^{4} \mathrm{~J}=3.1 \mathrm{~Hz}\right)$, $127.7\left({ }^{3} \mathrm{~J}=8.5 \mathrm{~Hz}\right), 125.8,115.0\left({ }^{2} \mathrm{~J}=21.4 \mathrm{~Hz}\right), 85.9,75.8,73.5$.<smiles>C#CC(O)(c1ccc(OC)cc1)c1ccc(C(O)(C#C)c2ccc(OC)cc2)cc1</smiles>

5c, 1,4-di[1-hydroxy-1-(p-methoxyphenyl)-prop-2-ynyl]benzene

The general procedure was followed using 1,4-di( $p$-methoxybenzoyl) benzene as starting material, pale-yellow solid was obtained in yield of $49 \%$.

mp $146-149^{\circ} \mathrm{C}$.

IR $\left(\mathrm{CHCl}_{3}\right): 3587,3305,3019,2959,2938,2840,1608,1585,1510,1465,1417,1304$, 1252, 1177, 1036, 987, 899, 833.

${ }^{1} \mathrm{H}$ NMR $\left(300 \mathrm{MHz}, \mathrm{CDCl}_{3}\right): \delta 7.55(\mathrm{~s}, 2 \mathrm{H}), 7.53-7.46(\mathrm{~m}, 2 \mathrm{H}), 6.88-6.81(\mathrm{~m}, 2 \mathrm{H}), 3.78$ (s, 3H), $2.85(\mathrm{~s}, 1 \mathrm{H}), 2.80(\mathrm{~s}, 1 \mathrm{H})$.

${ }^{13} \mathrm{C}$ NMR $\left(75 \mathrm{MHz}, \mathrm{CDCl}_{3}\right): \delta 159.0,143.9,136.3,127.2,125.8,113.5,86.4,75.3,73.7$, 55.3. 


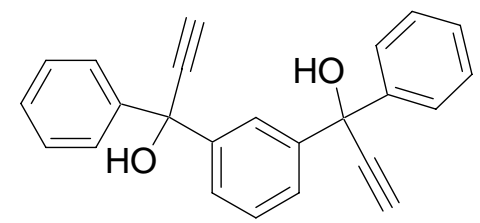

5'a, 1,3-di(1-hydroxy-1-phenyl-prop-2-ynyl)benzene

The general procedure was followed using 1,3-dibenzoyl benzene as starting material, beige solid was obtained in yield of $48 \%$.

mp $137-138.3^{\circ} \mathrm{C}$.

IR $\left(\mathrm{CHCl}_{3}\right): 3587,3306,3065,3018,2927,1600,1490,1450,1428,1325,1228,1164$, 1046, 1004, 987, 916, 878, 795.

${ }^{1} \mathrm{H}$ NMR $\left(300 \mathrm{MHz}, \mathrm{CDCl}_{3}\right): \delta 8.02(\mathrm{t}, \mathrm{J}=1.9 \mathrm{~Hz}, 1 \mathrm{H}), 7.61-7.54(\mathrm{~m}, 4 \mathrm{H}), 7.47(\mathrm{dd}, \mathrm{J}=$ 7.6, $1.9 \mathrm{~Hz}, 2 \mathrm{H}), 7.37-7.25(\mathrm{~m}, 7 \mathrm{H}), 2.87$ (s, 2H), 2.78 (s, 2H).

${ }^{13} \mathrm{C}$ NMR $\left(75 \mathrm{MHz}, \mathrm{CDCl}_{3}\right): \delta 144.3,144.0,128.2,127.7,125.8,125.6,123.3,86.2$, $75.6,74.3$.

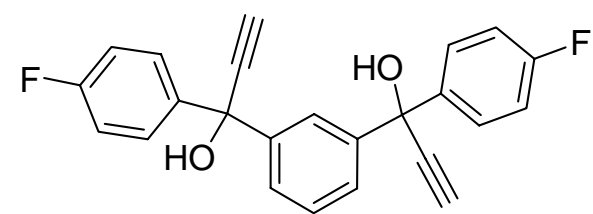

5'b, 1,3-di(1-hydroxy-1-p-methoxyphenyl-prop-2-ynyl)benzene

The general procedure was followed using 1,3-di(p-fluorobenzoyl) benzene as starting material, pale yellow gel was obtained in yield of $64 \%$.

mp 117-118.6 ${ }^{\circ} \mathrm{C}$.

IR $\left(\mathrm{CHCl}_{3}\right): 3587,3305,3018,2926,1604,1508,1482,1428,1410,1323,1232,1160$, $1054,1016,990,885,838$.

${ }^{1} \mathrm{H}$ NMR $\left(300 \mathrm{MHz}, \mathrm{CDCl}_{3}\right): \delta$ 7.94-7.90 (m, 1H), 7.56-7.50 (m, 4H), 7.49-7.44 (m, 2H), $7.30(\mathrm{dd}, \mathrm{J}=8.4,7.1 \mathrm{~Hz}, 1 \mathrm{H}), 7.04-6.95(\mathrm{~m}, 4 \mathrm{H}), 2.88(\mathrm{~s}, 2 \mathrm{H}), 2.87(\mathrm{~s}, 2 \mathrm{H})$.

${ }^{13} \mathrm{C}$ NMR $\left(75 \mathrm{MHz}, \mathrm{CDCl}_{3}\right): \delta 160.2\left({ }^{1} \mathrm{~J}=246.6 \mathrm{~Hz}\right), 144.3,139.9\left({ }^{4} \mathrm{~J}=3.9 \mathrm{~Hz}\right), 128.3$, $127.7\left({ }^{3} \mathrm{~J}=7.9 \mathrm{~Hz}\right), 125.7,123.2,115.0\left({ }^{2} \mathrm{~J}=22.0 \mathrm{~Hz}\right), 85.9,75.9,73.8$.

\section{General procedure for the preparation of bispyrans}

Bispropargyl alcohol $(0.25 \mathrm{mmol})$, naphthol $(0.55 \mathrm{mmol})$, PPTS $(0.05 \mathrm{mmol})$ and trimethyl orthoformate $(1 \mathrm{mmol})$ in 1,2-dichloroethane $(1.5 \mathrm{~mL})$ was refluxed for $4-24 \mathrm{~h}$, filtered through a pad of alumina while it was hot, and washed with 1,2-dichloroethane. The filtrate was concentrated, recrystalized from dichloromethane/hexane. The solid was collected by filtration. The mother liquid was concentrated and purified by chromatography (silica gel, dichloromethane : hexane $=1: 1$ ) and recrystalization (dichloromethane/hexane). All pyrans were obtained as a mixture of dl pair and meso. 


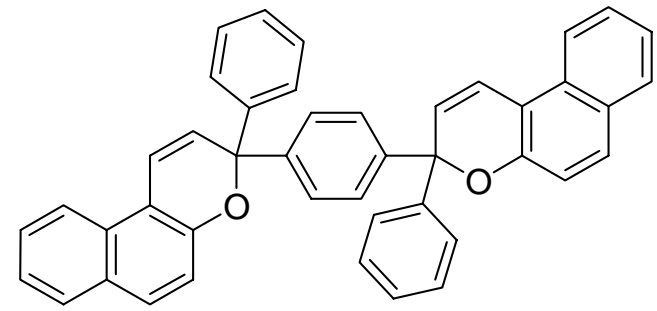

1a, 1,4-di-(3-phenyl-[3H]-naphtho[2,1-b]pyran-3-yl)-benzene

The general procedure was followed using 1,4-di(1-hydroxy-1-phenyl-prop-2ynyl)benzene and 2-naphthol as starting material and the mixture was refluxed for $5 \mathrm{~h}$, beige solid was obtained in yield of $81.6 \%$.

mp 282-284 ${ }^{\circ} \mathrm{C}$.

IR (KBr): 3056, 3022, 1632, 1588, 1514, 1460, 1447, 1386, 1242, 1218, 1083, 1006, 948, $805,750,728,698$.

${ }^{1} \mathrm{H}$ NMR (300 MHz, acetone): $\delta 8.04(\mathrm{~d}, \mathrm{~J}=9.0 \mathrm{~Hz}, 2 \mathrm{H}), 7.76(\mathrm{~d}, \mathrm{~J}=7.5 \mathrm{~Hz}, 2 \mathrm{H}), 7.73$ (d, J = 8.4 Hz, 2H), 7.55-7.40 (m, 12H), 7.35-7.18 (m, 10H), $6.47(\mathrm{~d}, \mathrm{~J}=10.0 \mathrm{~Hz}, 2 \mathrm{H})$.

${ }^{13} \mathrm{C}$ NMR $\left(75 \mathrm{MHz}, \mathrm{CDCl}_{3}\right): \delta 150.3,144.4,144.0,129.7,129.2,128.4,127.9,127.4$, $126.9,126.6,126.5,123.5,121.2,119.3,118.2,113.8,82.4$.

HRMS (MALDI) Calcd. for $\mathrm{C}_{44} \mathrm{H}_{30} \mathrm{O}_{2}: 613.2138\left(\mathrm{MNa}^{+}\right)$. Found: 613.2133 (56.96\%).<smiles>Fc1ccc(C2(c3ccc(C4(c5ccc(F)cc5)C=Cc5c(ccc6ccccc56)O4)cc3)C=Cc3c(ccc4ccccc34)O2)cc1</smiles>

1b, 1,4-di-(3-p-fluorophenyl-[3H]-naphtho[2,1-b]pyran-3-yl)-benzene

The general procedure was followed using 1,4-di[1-hydroxy-1-( $p$-fluorophenyl)-prop-2ynyl]benzene and 2-naphthol as starting material and the mixture was refluxed for $5 \mathrm{~h}$, beige solid was obtained in yield of $84 \%$.

mp 271-273 ${ }^{\circ} \mathrm{C}$.

IR (KBr): 3063, 1630, 1601, 1586, 1504, 1459, 1225, 1158, 1084, 1006, 952, 804, 827, $814,747,726$.

${ }^{1} \mathrm{H}$ NMR $\left(300 \mathrm{MHz}, \mathrm{CDCl}_{3}\right): \delta 7.94(\mathrm{~d}, \mathrm{~J}=8.4 \mathrm{~Hz}, 2 \mathrm{H}), 7.72(\mathrm{~d}, \mathrm{~J}=8.1 \mathrm{~Hz}, 2 \mathrm{H}), 7.65(\mathrm{~d}$, $\mathrm{J}=8.7 \mathrm{~Hz}, 2 \mathrm{H}), 7.51-7.40(\mathrm{~m}, 6 \mathrm{H}), 7.40(\mathrm{~s}, 4 \mathrm{H}), 7.36-7.29(\mathrm{~m}, 2 \mathrm{H}), 7.29(\mathrm{~d}, \mathrm{~J}=10.0 \mathrm{~Hz}$, $2 \mathrm{H}), 7.15(\mathrm{~d}, \mathrm{~J}=8.7 \mathrm{~Hz}, 2 \mathrm{H}), 7.02-6.90(\mathrm{~m}, 4 \mathrm{H}), 6.18(\mathrm{~d}, \mathrm{~J}=10.0 \mathrm{~Hz}, 2 \mathrm{H})$.

${ }^{13} \mathrm{C}$ NMR $\left(75 \mathrm{MHz}, \mathrm{CDCl}_{3}\right): \delta 162.0\left({ }^{1} \mathrm{~J}=246.0 \mathrm{~Hz}\right), 150.1,144.0,140.1\left({ }^{4} \mathrm{~J}=3.1 \mathrm{~Hz}\right)$, 129.9, 129.6, 129.2, $128.8\left({ }^{3} \mathrm{~J}=7.9 \mathrm{~Hz}\right), 128.4,127.1,126.6,123.6,121.2,119.6,118.1$, $114.8\left({ }^{2} \mathrm{~J}=21.4 \mathrm{~Hz}\right), 113.8,81.9$.

HRMS (MALDI) Calcd. for $\mathrm{C}_{44} \mathrm{H}_{28} \mathrm{~F}_{2} \mathrm{O}_{2}: 627.2130\left(\mathrm{MH}^{+}\right)$. Found: 627.2137 (100\%). 
<smiles>COc1ccc(C2(c3ccc(C4(c5ccc(OC)cc5)C=Cc5c(ccc6ccccc56)O4)cc3)C=Cc3c(ccc4ccccc34)O2)cc1</smiles>

1c, 1,4-di-(3-p-methoxyphenyl-[3H]-naphtho[2,1-b]pyran-3-yl)-benzene

The general procedure was followed using 1,4-di[1-hydroxy-1-( $p$-methoxyphenyl)-prop2-ynyl]benzene and 2-naphthol as starting material and the mixture was refluxed for $5 \mathrm{~h}$, beige solid was obtained in yield of $88 \%$.

$\operatorname{mp} 277-279{ }^{\circ} \mathrm{C}$.

IR (KBr): 3060, 2931, 2835, 1629, 1606, 1589, 1508, 1462, 1254, 1228, 1172, 1084 , $1004,958,824,812,745$.

${ }^{1} \mathrm{H}$ NMR (300 MHz, $\left.\mathrm{CDCl}_{3}\right): \delta 7.94(\mathrm{~d}, \mathrm{~J}=8.7 \mathrm{~Hz}, 2 \mathrm{H}), 7.70(\mathrm{~d}, \mathrm{~J}=7.8 \mathrm{~Hz}, 2 \mathrm{H}), 7.63(\mathrm{~d}$, $\mathrm{J}=9.0 \mathrm{~Hz}, 2 \mathrm{H}), 7.49-7.41(\mathrm{~m}, 2 \mathrm{H}), 7.41(\mathrm{~s}, 4 \mathrm{H}), 7.40-7.34(\mathrm{~m}, 4 \mathrm{H}), 7.34-7.29(\mathrm{~m}, 2 \mathrm{H})$, $7.27(\mathrm{~d}, \mathrm{~J}=10.0 \mathrm{~Hz}, 2 \mathrm{H}), 7.15(\mathrm{~d}, \mathrm{~J}=9.0 \mathrm{~Hz}, 2 \mathrm{H}), 6.85-6.76(\mathrm{~m}, 4 \mathrm{H}), 6.20(\mathrm{~d}, \mathrm{~J}=10.0$ $\mathrm{Hz}, 2 \mathrm{H}), 3.75$ (s, 6H).

${ }^{13} \mathrm{C}$ NMR $\left(75 \mathrm{MHz}, \mathrm{CDCl}_{3}\right): \delta 158.7,150.3,144.1,136.5,129.6,129.1,128.3,127.6$, 123.4, 121.2, 119.1, 118.2, 113.8, 113.3, 82.2, 55.2.

HRMS (MALDI) Calcd. for $\mathrm{C}_{46} \mathrm{H}_{34} \mathrm{O}_{4}: 651.2530\left(\mathrm{MH}^{+}\right)$. Found: 651.2537 (100\%).

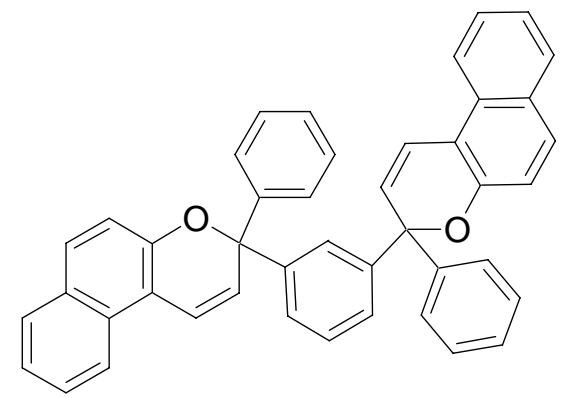

1'a, 1,3-di-(3-phenyl-[3H]-naphtho[2,1-b]pyran-3-yl)-benzene

The general procedure was followed using 1,3-di(1-hydroxy-1-phenyl-prop-2ynyl)benzene and 2-naphthol as starting material and the mixture was refluxed for $4 \mathrm{~h}$, beige solid was obtained in yield of $74 \%$.

mp $163-166^{\circ} \mathrm{C}$.

IR (KBr): 3055, 1636, 1602, 1582, 1507, 1458, 1376, 1226, 1185, 1158, 1090, 1072, $1005,946,880,834,815,772,726,668$.

${ }^{1} \mathrm{H}$ NMR $\left(300 \mathrm{MHz}, \mathrm{CD}_{2} \mathrm{Cl}_{2}\right): \delta 7.93(\mathrm{~d}, \mathrm{~J}=8.4 \mathrm{~Hz}, 2 \mathrm{H}), 7.76-7.66(\mathrm{~m}, 3 H), 7.61(\mathrm{~d}, \mathrm{~J}=$ $9.0 \mathrm{~Hz}, 2 \mathrm{H}), 7.51-7.43(\mathrm{~m}, 2 \mathrm{H}), 7.41-7.18(\mathrm{~m}, 17 \mathrm{H}), 7.09(\mathrm{~d}, \mathrm{~J}=8.7 \mathrm{~Hz}, 1 \mathrm{H}), 7.08(\mathrm{~d}, \mathrm{~J}=$ $8.7 \mathrm{~Hz}, 1 \mathrm{H}), 6.27(\mathrm{~d}, \mathrm{~J}=10.0 \mathrm{~Hz}, 1 \mathrm{H}), 6.25(\mathrm{~d}, \mathrm{~J}=10.0 \mathrm{~Hz}, 1 \mathrm{H})$,

${ }^{13} \mathrm{C}$ NMR $\left(75 \mathrm{MHz}, \mathrm{CDCl}_{3}\right): \delta 150.3,150.3,144.5,144.5,144.2,144.0,129.6,129.6$, 129.2, 128.3, 127.9, 127.6, 127.6, 127.6, 127.4, 127.3, 126.9, 126.8, 126.4, 126.2, 126.0, 126.0, 125.6, 123.4, 123.4, 121.2, 121.2, 119.5, 119.4, 118.2, 118.2, 114.0, 114.0, 82.5. HRMS (MALDI) Calcd for $\mathrm{C}_{44} \mathrm{H}_{30} \mathrm{O}_{2}: 613.2138\left(\mathrm{MNa}^{+}\right)$. Found: 613.2136 (38.50\%). 


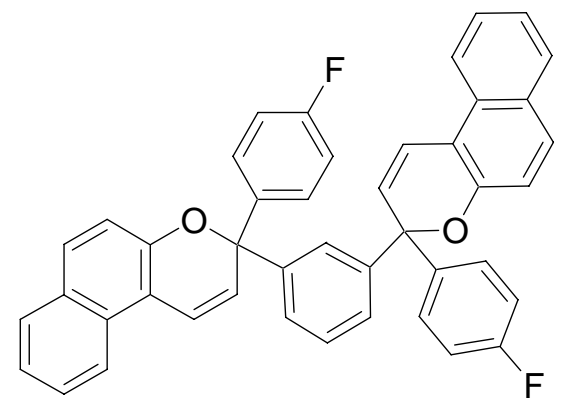

1'b, 1,3-di-(3-p-fluorophenyl-[3H]-naphtho[2,1-b]pyran-3-yl)-benzene

The general procedure was followed using 1,4-di[1-hydroxy-1-( $p$-fluorophenyl)-prop-2ynyl]benzene and 2-naphthol as starting material and the mixture was refluxed for $6 \mathrm{~h}$, beige solid was obtained in yield of $73 \%$.

mp 203-205 ${ }^{\circ} \mathrm{C}$.

IR (KBr): 3060, 1631, 1602, 1587, 1507, 1459, 1384, 3284, 1269, 1238, 1202, 1178, $1158,1087,1021,950,833,814,731,706$.

${ }^{1} \mathrm{H}$ NMR $\left(300 \mathrm{MHz}, \mathrm{CDCl}_{3}\right): \delta 7.91(\mathrm{~d}, \mathrm{~J}=8.1 \mathrm{~Hz}, 2 \mathrm{H}), 7.72(\mathrm{~d}, \mathrm{~J}=7.8 \mathrm{~Hz}, 2 \mathrm{H}), 7.59(\mathrm{~d}$, $\mathrm{J}=8.7 \mathrm{~Hz}, 2 \mathrm{H}), 7.62-7.54(\mathrm{~m}, 1 \mathrm{H}), 7.51-7.42(\mathrm{~m}, 2 \mathrm{H}), 7.39-7.20(\mathrm{~m}, 11 \mathrm{H}), 7.05(\mathrm{~d}, \mathrm{~J}=$ $8.7 \mathrm{~Hz}, 1 \mathrm{H}), 7.04(\mathrm{~d}, \mathrm{~J}=8.7 \mathrm{~Hz}, 1 \mathrm{H}), 7.74-6.84(\mathrm{~m}, 4 \mathrm{H}), 6.15(\mathrm{~d}, \mathrm{~J}=10.0 \mathrm{~Hz}, 1 \mathrm{H}), 6.14$ $(\mathrm{d}, \mathrm{J}=10.0 \mathrm{~Hz}, 1 \mathrm{H})$.

${ }^{13} \mathrm{C}$ NMR $\left(75 \mathrm{MHz}, \mathrm{CDCl}_{3}\right): \delta 162.0\left({ }^{1} \mathrm{~J}=246.6 \mathrm{~Hz}\right), 150.3,144.3,144.1,140.42\left({ }^{4} \mathrm{~J}=\right.$ $3.1 \mathrm{~Hz}), 140.37\left({ }^{4} \mathrm{~J}=3.1 \mathrm{~Hz}\right), 129.9,129.7,129.3,128.9\left({ }^{3} \mathrm{~J}=7.9 \mathrm{~Hz}\right), 128.8,128.5$, $127.9,127.9,127.4,126.7,126.3,126.1,125.9,125.5,123.7,121.3,119.9,119.8,118.2$, $118.2,114.8\left({ }^{2} \mathrm{~J}=21.4 \mathrm{~Hz}\right), 114.1,114.1,82.1$.

HRMS (MALDI) Calcd. for $\mathrm{C}_{44} \mathrm{H}_{28} \mathrm{~F}_{2} \mathrm{O}_{2}: 649.1950\left(\mathrm{MNa}^{+}\right)$. Found: 649.1958 (100\%).

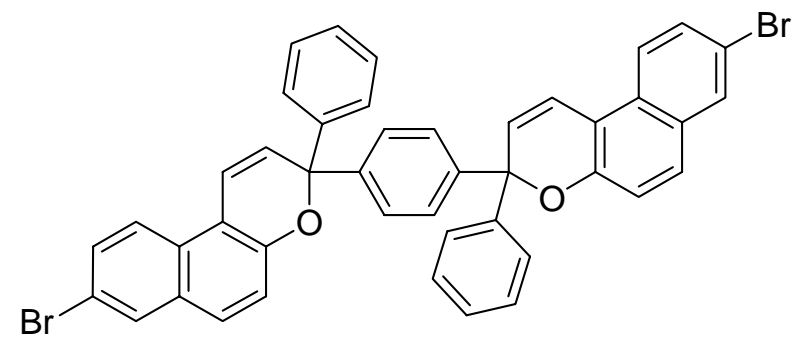

2a, 1,4-di-(8-bromo-3-phenyl-[3H]-naphtho[2,1-b]pyran-3-yl)-benzene

The general procedure was followed using 1,4-di(1-hydroxy-1-phenyl-prop-2ynyl)benzene and 6-bromo-2-naphthol as starting material and the mixture was refluxed for $6.5 \mathrm{~h}$, pale-yellow solid was obtained in yield of $94 \%$. mp $267-269^{\circ} \mathrm{C}$.

IR (KBr): 3056, 1630, 1582, 1498, 1446, 1376, 1247, 1213, 1183, 1091, 1069, 1004, 938, $878,813,750,700$.

${ }^{1} \mathrm{H}$ NMR $\left(300 \mathrm{MHz}, \mathrm{CDCl}_{3}\right): \delta 7.84(\mathrm{~d}, \mathrm{~J}=1.9 \mathrm{~Hz}, 2 \mathrm{H}), 7.78(\mathrm{~d}, \mathrm{~J}=8.7 \mathrm{~Hz}, 2 \mathrm{H}), 7.53(\mathrm{~d}$, $\mathrm{J}=8.7 \mathrm{~Hz}, 2 \mathrm{H}), 7.52-7.47(\mathrm{~m}, 2 \mathrm{H}), 7.46-7.41(\mathrm{~m}, 4 \mathrm{H}), 7.40(\mathrm{~s}, 4 \mathrm{H}), 7.34-7.22(\mathrm{~m}, 6 \mathrm{H})$, $7.21(\mathrm{~d}, \mathrm{~J}=9.9 \mathrm{~Hz}, 2 \mathrm{H}), 7.17(\mathrm{~d}, \mathrm{~J}=8.4 \mathrm{~Hz}, 2 \mathrm{H}), 6.24(\mathrm{~d}, \mathrm{~J}=9.9 \mathrm{~Hz}, 2 \mathrm{H})$.

${ }^{13} \mathrm{C}$ NMR $\left(75 \mathrm{MHz}, \mathrm{CDCl}_{3}\right): \delta 150.4,144.2,143.9,130.2,130.1,129.6,128.7,128.0$, 127.9, 127.5, 126.8, 126.7, 122.9, 119.2, 118.9, 117.2, 113.9, 82.5.

HRMS (MALDI) Calcd. for $\mathrm{C}_{44} \mathrm{H}_{28} \mathrm{Br}_{2} \mathrm{O}_{2}: 771.0331\left(\mathrm{MNa}^{+}\right)$. Found: 771.0330 (47.04\%). 
<smiles>Fc1ccc(C2(c3ccc(C4(c5ccc(F)cc5)C=Cc5c(ccc6cc(Br)ccc56)O4)cc3)C=Cc3c(ccc4cc(Br)ccc34)O2)cc1</smiles>

2b, 1,4-di-(3-p-fluorophenyl-[3H]-naphtho[2,1-b]pyran-3-yl)-benzene

The general procedure was followed using 1,4-di[1-hydroxy-1-( $p$-fluorophenyl)-prop-2ynyl]benzene and 6-bromo-2-naphthol as starting material and the mixture was refluxed for $5.5 \mathrm{~h}$, beige solid was obtained in yield of $78.4 \%$.

mp 270-272 ${ }^{\circ} \mathrm{C}$.

IR (KBr): 3063, 1636, 1602, 1582, 1507, 1458, 1226, 1158, 1090, 1072, 1005, 946, 880, $834815,772$.

${ }^{1} \mathrm{H}$ NMR (300 MHz, $\left.\mathrm{CDCl}_{3}\right): \delta 7.84(t, \mathrm{~J}=1.9 \mathrm{~Hz}, 2 \mathrm{H}), 7.77(\mathrm{dd}, \mathrm{J}=9.0,2.2 \mathrm{~Hz}, 2 \mathrm{H})$, $7.53(\mathrm{~d}, \mathrm{~J}=8.4 \mathrm{~Hz}, 2 \mathrm{H}), 7.50(\mathrm{dt}, \mathrm{J}=9.0,2.2 \mathrm{~Hz}, 2 \mathrm{H}), 7.45-7.38(\mathrm{~m}, 8 \mathrm{H}), 7.21(\mathrm{~d}, \mathrm{~J}=$ $10.0 \mathrm{~Hz}, 2 \mathrm{H}), 7.15(\mathrm{~d}, \mathrm{~J}=8.7 \mathrm{~Hz}, 2 \mathrm{H}), 7.03-6.92(\mathrm{~m}, 4 \mathrm{H}), 6.19$ (d, J = $10.0 \mathrm{~Hz}, 2 \mathrm{H})$.

${ }^{13} \mathrm{C}$ NMR $\left(75 \mathrm{MHz}, \mathrm{CDCl}_{3}\right): \delta 162.0\left({ }^{1} \mathrm{~J}=246.0 \mathrm{~Hz}\right), 160.3,150.3,143.9,139.9\left({ }^{4} \mathrm{~J}=\right.$ $3.1 \mathrm{~Hz}), 130.3,130.2,129.7,128.9,128.7\left({ }^{3} \mathrm{~J}=7.9 \mathrm{~Hz}\right), 128.1,127.6,126.6,122.9,119.2$, $119.1,117.3,114.9\left({ }^{2} \mathrm{~J}=21.4 \mathrm{~Hz}\right), 113.9,82.0$.

HRMS (MALDI) Calcd. for $\mathrm{C}_{44} \mathrm{H}_{26} \mathrm{Br}_{2} \mathrm{~F}_{2} \mathrm{O}_{2}: 785.0323\left(\mathrm{MH}^{+}\right)$. Found: 785.0331 (100\%).

${ }^{19} \mathrm{~F} \mathrm{NMR}\left(300 \mathrm{MHz}, \mathrm{CDCl}_{3}\right): \delta-114.10$<smiles>COc1ccc(C2(c3ccc(C4(c5ccc(OC)cc5)C=Cc5c(ccc6cc(Br)ccc56)O4)cc3)C=Cc3c(ccc4cc(Br)ccc34)O2)cc1</smiles>

2c, 1,4-di-(8-bromo-3-p-methoxyphenyl-[3H]-naphtho[2,1-b]pyran-3-yl)-benzene

The general procedure was followed using 1,4-di[1-hydroxy-1-( $p$-methoxyphenyl)-prop2-ynyl]benzene and 6-bromo-2-naphthol as starting material and the mixture was refluxed for $5 \mathrm{~h}$, pale-yellow solid was obtained in yield of $93 \%$.

mp 259-262 ${ }^{\circ} \mathrm{C}$.

IR (KBr): 2930, 2834, 1629, 1608, 1583, 1510, 1458, 1304, 1251, 1226, 1173, 1089, 1071, 1002, 836, 808.

${ }^{1} \mathrm{H}$ NMR $\left(300 \mathrm{MHz}, \mathrm{CDCl}_{3}\right): \delta 7.81(t, \mathrm{~J}=1.9 \mathrm{~Hz}, 2 \mathrm{H}), 7.75(\mathrm{dd}, \mathrm{J}=9.0,3.7 \mathrm{~Hz}, 2 \mathrm{H})$, $7.51(\mathrm{dd}, \mathrm{J}=9.0,1.5 \mathrm{~Hz}, 2 \mathrm{H}), 7.47(t d, \mathrm{~J}=8.7,2.3 \mathrm{~Hz}, 2 \mathrm{H}), 7.40(\mathrm{~s}, 4 \mathrm{H}), 7.38-7.30(\mathrm{~m}$, $4 \mathrm{H}), 7.17(\mathrm{~d}, \mathrm{~J}=10.0 \mathrm{~Hz}, 2 \mathrm{H}), 7.15(\mathrm{~d}, \mathrm{~J}=8.7 \mathrm{~Hz}, 2 \mathrm{H}), 6.85-6.76(\mathrm{~m}, 4 \mathrm{H}), 6.21(\mathrm{~d}, \mathrm{~J}=$ $10.0 \mathrm{~Hz}, 2 \mathrm{H}), 3.75(\mathrm{~s}, 6 \mathrm{H})$. 
${ }^{13} \mathrm{C}$ NMR $\left(75 \mathrm{MHz}, \mathrm{CDCl}_{3}\right): \delta 158.8,150.4,144.0,136.3,130.2,130.1,129.5,128.6$, 128.3, 128.0, 126.5, 122.9, 119.2, 118.8, 117.1, 113.9, 113.3, 82.3, 55.2.

HRMS (MALDI) Calcd. for $\mathrm{C}_{46} \mathrm{H}_{32} \mathrm{Br}_{2} \mathrm{O}_{4}: 807.0740\left(\mathrm{MH}^{+}\right)$. Found: 807.0735 (100\%).<smiles>Brc1ccc2c3c(ccc2c1)OC(c1ccccc1)(c1cccc(C2(c4ccccc4)C=Cc4c(ccc5cc(Br)ccc45)O2)c1)C=C3</smiles>

2'a, 1,3-di-(8-bromo-3-phenyl-[3H]-naphtho[2,1-b]pyran-3-yl)-benzene

The general procedure was followed using 1,3-di(1-hydroxy-1-phenyl-prop-2ynyl)benzene and 6-bromo-2-naphthol as starting material and the mixture was refluxed for $6.5 \mathrm{~h}$, beige solid was obtained in yield of $81 \%$.

mp $142-146^{\circ} \mathrm{C}$.

IR (KBr): 3056, 2922, 1631, 1583, 1500, 1446, 1377, 1356, 1326, 1264, 1236, 1213 , 1082, 1153, 1090, 1070, 1007, 987, 876, 807, 772, 764, 731, 706, 698.

${ }^{1} \mathrm{H}$ NMR $\left(300 \mathrm{MHz}, \mathrm{CDCl}_{3}\right): \delta 7.85(\mathrm{~d}, \mathrm{~J}=1.8 \mathrm{~Hz}, 2 \mathrm{H}), 7.75(\mathrm{~d}, \mathrm{~J}=8.7 \mathrm{~Hz}, 2 \mathrm{H}), 7.66-$ $7.58(\mathrm{~m}, 1 \mathrm{H}), 7.54-7.44(\mathrm{~m}, 4 \mathrm{H}), 7.39-7.30(\mathrm{~m}, 6 \mathrm{H}), 7.30-7.20(\mathrm{~m}, 7), 7.18(\mathrm{~d}, \mathrm{~J}=10.0$ $\mathrm{Hz}, 1 \mathrm{H}), 7.17(\mathrm{~d}, \mathrm{~J}=10.0 \mathrm{~Hz}, 1 \mathrm{H}), 7.06(\mathrm{~d}, \mathrm{~J}=8.7 \mathrm{~Hz}, 2 \mathrm{H}), 6.20(\mathrm{~d}, \mathrm{~J}=10.0 \mathrm{~Hz}, 1 \mathrm{H})$, $6.19(\mathrm{~d}, \mathrm{~J}=10.0 \mathrm{~Hz}, 1 \mathrm{H})$.

${ }^{13} \mathrm{C}$ NMR (75 MHz, $\left.\mathrm{CDCl}_{3}\right): \delta 150.4,144.2,144.2,144.1,143.9,130.3,130.2,129.6$, 128.6, 128.0, 128.0, 127.8, 127.5, 127.5, 126.8, 126.8, 126.3, 126.1, 125.8, 125.5, 123.0, 119.2, 119.1, 119.0, 117.2, 114.1, 114.1, 82.6.

HRMS (MALDI) Calcd. for $\mathrm{C}_{44} \mathrm{H}_{28} \mathrm{Br}_{2} \mathrm{O}_{2}$ : 771.0331 ( $\mathrm{MNa}^{+}$). Found: 771.0328 (74.45\%).<smiles>Fc1ccc(C2(c3cccc(C4(c5ccc(F)cc5)C=Cc5c(ccc6cc(Br)ccc56)O4)c3)C=Cc3c(ccc4cc(Br)ccc34)O2)cc1</smiles>

2'b, 1,3-di-(8-bromo-3-p-fluorophenyl-[3H]-naphtho[2,1-b]pyran-3-yl)-benzene

The general procedure was followed using 1,3- di[1-hydroxy-1-( $p$-fluorophenyl)-prop-2ynyl]benzene and 6-bromo-2-naphthol as starting material and the mixture was refluxed for $8 \mathrm{~h}$, white solid was obtained in yield of $86 \%$.

mp $187-191^{\circ} \mathrm{C}$. 
IR (KBr): 3056, 2921, 1633, 1601, 1582, 1507, 1458, 1376, 1356, 1325, 1264, 1228, 1158, 1089, 1071, 1008, 951, 879, 840, 814, 775, 743, 698.

${ }^{1} \mathrm{H}$ NMR $\left(300 \mathrm{MHz}, \mathrm{CDCl}_{3}\right): \delta 7.87(\mathrm{~d}, \mathrm{~J}=2.1 \mathrm{~Hz}, 2 \mathrm{H}), 7.76(\mathrm{dd}, \mathrm{J}=9.0,1.6 \mathrm{~Hz}, 2 \mathrm{H})$, 7.57-7.46 (m, 5H), 7.37-7.22 (m, 7H), $7.19(\mathrm{~d}, \mathrm{~J}=10.0 \mathrm{~Hz}, 1 \mathrm{H}), 7.18(\mathrm{~d}, \mathrm{~J}=10.0 \mathrm{~Hz}$, $1 \mathrm{H}), 7.04(\mathrm{~d}, \mathrm{~J}=9.0 \mathrm{~Hz}, 1 \mathrm{H}), 7.03(\mathrm{~d}, \mathrm{~J}=9.0 \mathrm{~Hz}, 1 \mathrm{H}), 6.97-6.86(\mathrm{~m}, 4 \mathrm{H}), 6.15(\mathrm{~d}, \mathrm{~J}=$ $10.0 \mathrm{~Hz}, 1 \mathrm{H}), 6.14(\mathrm{~d}, \mathrm{~J}=10.0 \mathrm{~Hz}, 1 \mathrm{H})$.

${ }^{13} \mathrm{C}$ NMR $\left(75 \mathrm{MHz}, \mathrm{CDCl}_{3}\right): \delta 161.9\left({ }^{1} \mathrm{~J}=246.6 \mathrm{~Hz}\right), 150.2,150.2,144.1,143.9,139.99$ $\left({ }^{4} \mathrm{~J}=2.8 \mathrm{~Hz}\right), 139.95\left({ }^{4} \mathrm{~J}=2.8 \mathrm{~Hz}\right), 130.3,130.2,129.7,128.8,128.7\left({ }^{3} \mathrm{~J}=7.9 \mathrm{~Hz}\right), 128.0$, $128.0,127.9,127.7,126.3,126.1,125.6,125.2,123.0,119.4,119.3,119.1,117.4,114.9$ $\left({ }^{2} \mathrm{~J}=21.4 \mathrm{~Hz}\right), 114.1,114.0,82.2$.

HRMS (MALDI) Calcd. for $\mathrm{C}_{44} \mathrm{H}_{26} \mathrm{Br}_{2} \mathrm{~F}_{2} \mathrm{O}_{2}: 785.0323\left(\mathrm{MH}^{+}\right)$. Found: $785.0332(100 \%)$.<smiles>CCOC(=O)c1c2c(c3ccccc3c1-c1ccccc1)OC(c1ccccc1)(c1ccccc1)C=Cc1c(C(=O)OCC)c(-c3ccccc3)c3ccccc3c1-2</smiles>

3a, 1,4-di-(2,6-diphenyl-5-ethoxycarbonyl-[2H]-naphtho[1,2-b]pyran-2-yl)-benzene The general procedure was followed using 1,4-di(1-hydroxy-1-phenyl-prop-2ynyl)benzene and 4-Hydroxy-1-phenyl-naphthoic acid ethyl ester (3 equiv) as starting material and the mixture was refluxed for $5.5 \mathrm{~h}$, pale pink solid was obtained in yield of $71 \%$.

mp $283-285^{\circ} \mathrm{C}$.

IR (KBr): 3048, 2979, 1730, 1645, 1599, 1504, 1448, 1437, 1393, 1372, 1294, 1258, $1223,1167,1148,1113,1044,998,834,773,756,699$.

${ }^{1} \mathrm{H}$ NMR $\left(300 \mathrm{MHz}, \mathrm{CDCl}_{3}\right): \delta 8.42(\mathrm{~d}, \mathrm{~J}=8.1 \mathrm{~Hz}, 2 \mathrm{H}), 7.58-7.45(\mathrm{~m}, 12 \mathrm{H}), 7.45-7.22$ $(\mathrm{m}, 18 \mathrm{H}), 6.81(\mathrm{~d}, \mathrm{~J}=10.0 \mathrm{~Hz}, 2 \mathrm{H}), 6.23(\mathrm{~d}, \mathrm{~J}=10.0 \mathrm{~Hz}, 2 \mathrm{H}), 3.98(\mathrm{q}, \mathrm{J}=7.2 \mathrm{~Hz}, 4 \mathrm{H})$, $0.87(\mathrm{t}, \mathrm{J}=7.2 \mathrm{~Hz}, 6 \mathrm{H})$.

${ }^{13} \mathrm{C}$ NMR $\left(75 \mathrm{MHz}, \mathrm{CDCl}_{3}\right): \delta 168.2,147.2,144.4,144.2,138.1,132.7,130.8,130.4$, $128.6,128.1,127.8,127.7,127.6,127.3,126.8,126.7,126.7,126.4,124.9,122.0,120.7$, $111.8,83.0,61.1,13.7$.

HRMS (MALDI) Calcd. for $\mathrm{C}_{62} \mathrm{H}_{46} \mathrm{O}_{6}: 909.3187\left(\mathrm{MNa}^{+}\right)$. Found: 909.3180 (100\%).<smiles></smiles> 
3b, 1,4-di-2-( $p$-fluorophenyl-6-phenyl-5-ethoxycarbonyl-[2H]-naphtho[1,2-b]pyran-2yl)-benzene

The general procedure was followed using 1,4-di[1-hydroxy-1-( $p$-fluorophenyl)-prop-2ynyl]benzene and 4-Hydroxy-1-phenyl-naphthoic acid ethyl ester (3 equiv) as starting material and the mixture was refluxed for $24 \mathrm{~h}$, pale pink solid was obtained in yield of $65 \%$.

mp $287-289^{\circ} \mathrm{C}$.

IR (KBr): 3048, 2981, 1730, 1644, 1601, 1506, 1437, 1372, 1291, 1258, 1226, 1204 , $1158,1043,840,827,773,700,661$.

${ }^{1} \mathrm{H} \mathrm{NMR}\left(300 \mathrm{MHz}, \mathrm{CDCl}_{3}\right): \delta 8.40(\mathrm{~d}, \mathrm{~J}=8.1 \mathrm{~Hz}, 2 \mathrm{H}), 7.62-7.29(\mathrm{~m}, 24 \mathrm{H}), 7.08-6.96$ $(\mathrm{m}, 4 \mathrm{H}), 6.85(\mathrm{~d}, \mathrm{~J}=10.0 \mathrm{~Hz}, 2 \mathrm{H}), 6.20(\mathrm{~d}, \mathrm{~J}=10.0 \mathrm{~Hz}, 2 \mathrm{H}), 4.01(\mathrm{q}, \mathrm{J}=7.2 \mathrm{~Hz}, 4 \mathrm{H})$, $0.89(\mathrm{t}, \mathrm{J}=7.2 \mathrm{~Hz}, 6 \mathrm{H})$.

${ }^{13} \mathrm{C}$ NMR $\left(75 \mathrm{MHz}, \mathrm{CDCl}_{3}\right): \delta 168.2,161.0\left({ }^{1} \mathrm{~J}=246.6 \mathrm{~Hz}\right), 147.0,144.1,140.18\left({ }^{4} \mathrm{~J}=\right.$ $3.1 \mathrm{~Hz}), 140.16\left({ }^{4} \mathrm{~J}=3.1 \mathrm{~Hz}\right), 137.9,132.7,131.0,130.4,130.3,128.8\left({ }^{3} \mathrm{~J}=7.3 \mathrm{~Hz}\right)$, 128.6, 127.9, 127.8, 127.4, 127.3, 127.0, 126.9, 126.6, 126.6, 126.5, 124.8, 121.8, 121.0, $115.0\left({ }^{2} \mathrm{~J}=21.4 \mathrm{~Hz}\right), 111.8,111.8,82.6,61.1,13.7$.

HRMS (MALDI) Calcd. for $\mathrm{C}_{62} \mathrm{H}_{44} \mathrm{~F}_{2} \mathrm{O}_{6}: 945.2998\left(\mathrm{MNa}^{+}\right)$. Found: 945.3008 .

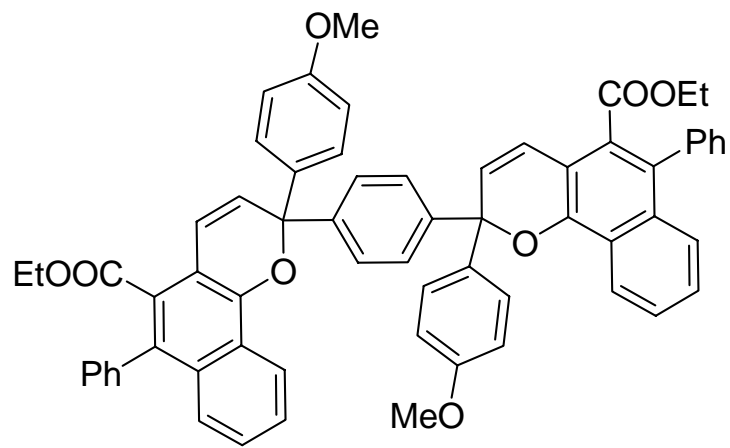

3c, 1,4-di-(2-p-methoxyphenyl-6-phenyl-5-ethoxycarbonyl-[2H]-naphtho[1,2-b]pyran-2yl)-benzene

The general procedure was followed using 1,4-di[1-hydroxy-1-(p-methoxyphenyl)-prop2-ynyl]benzene and 4-Hydroxy-1-phenyl-naphthoic acid ethyl ester as starting material and the mixture was refluxed for $20 \mathrm{~h}$, pink solid was obtained in yield of $84 \%$.

mp $254-256{ }^{\circ} \mathrm{C}$.

IR (KBr): 3072, 2926, 2836, 1721, 1608, 1508, 1445, 1370, 1308, 1297, 1255, 1227, $1178,1040,934,836,828,766,662$.

${ }^{1} \mathrm{H}$ NMR $\left(300 \mathrm{MHz}, \mathrm{CDCl}_{3}\right): \delta 8.39(\mathrm{dd}, \mathrm{J}=7.8,1.6 \mathrm{~Hz}, 2 \mathrm{H}), 7.54-7.28(\mathrm{~m}, 24 \mathrm{H}), 6.88-$ $6.81(\mathrm{~m}, 4 \mathrm{H}), 6.79(\mathrm{~d}, \mathrm{~J}=10.0 \mathrm{~Hz}, 2 \mathrm{H}), 6.20(\mathrm{~d}, \mathrm{~J}=10.0 \mathrm{~Hz}, 2 \mathrm{H}), 3.98(\mathrm{q}, \mathrm{J}=7.2 \mathrm{~Hz}$, $4 \mathrm{H}), 3.77(\mathrm{~s}, 6 \mathrm{H}), 0.87(\mathrm{t}, \mathrm{J}=7.2 \mathrm{~Hz}, 6 \mathrm{H})$.

${ }^{13} \mathrm{C}$ NMR $\left(75 \mathrm{MHz}, \mathrm{CDCl}_{3}\right): \delta 168.3,158.9,147.3,144.3,138.1,136.6,132.7,130.7$, $130.4,130.4,128.6,128.3,128.3,127.9,127.8,127.8,127.3,126.9,126.5,126.4,124.9$, $122.0,120.6,113.4,111.8,82.9,61.1,55.2,13.7$.

HRMS (MALDI) Calcd. for $\mathrm{C}_{64} \mathrm{H}_{50} \mathrm{O}_{8}: 969.3398\left(\mathrm{MNa}^{+}\right)$. Found: 969.3389 (72.2\%). 


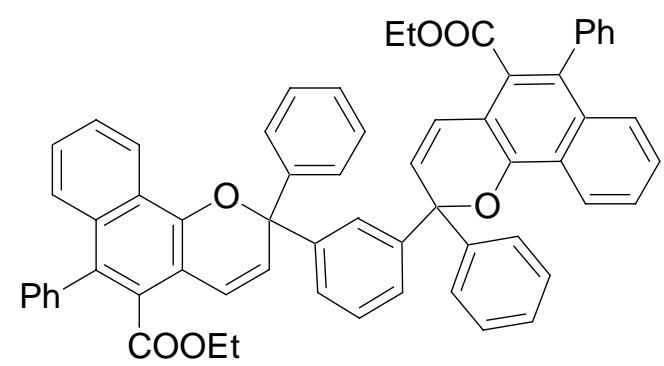

3'a, 1,3-di-(2,6-diphenyl-5-ethoxycarbonyl-[2H]-naphtho[1,2-b]pyran-2-yl)-benzene

The general procedure was followed using 1,3-di(1-hydroxy-1-phenyl-prop-2ynyl)benzene and 4-Hydroxy-1-phenyl-naphthoic acid ethyl ester as starting material and the mixture was refluxed for $24 \mathrm{~h}$, pale orange foam was obtained in yield of $69 \%$. mp 259-262 ${ }^{\circ} \mathrm{C}$.

${ }^{1} \mathrm{H}$ NMR $\left(300 \mathrm{MHz}, \mathrm{CDCl}_{3}\right): \delta 8.32-8.25(\mathrm{~m}, 2 \mathrm{H}), 7.83-7.72(\mathrm{~m}, 1 \mathrm{H}), 7.54-7.37(\mathrm{~m}$, $17 \mathrm{H}), 7.37-7.19(\mathrm{~m}, 12 \mathrm{H}), 6.79(\mathrm{~d}, \mathrm{~J}=10.0 \mathrm{~Hz}, 1 \mathrm{H}), 7.78(\mathrm{~d}, \mathrm{~J}=10.0 \mathrm{~Hz}, 1 \mathrm{H}), 6.18(\mathrm{~d}, \mathrm{~J}$ $=10.0 \mathrm{~Hz}, 1 \mathrm{H}), 6.17(\mathrm{~d}, \mathrm{~J}=10.0 \mathrm{~Hz}, 1 \mathrm{H}), 3.98(\mathrm{q}, \mathrm{J}=7.2 \mathrm{~Hz}, 4 \mathrm{H}), 0.87$ (t, J = 7.2 Hz, $6 \mathrm{H})$.

${ }^{13} \mathrm{C}$ NMR $\left(75 \mathrm{MHz}, \mathrm{CDCl}_{3}\right): \delta 168.2,147.2,144.6,144.5,144.5,144.5,138.1,132.7$, 132.6, 130.8, 130.4, 128.6, 128.6, 128.0, 127.9, 127.8, 127.5, 127.3, 126.8, 126.8, 126.7, $126.5,126.4,126.1,126.1,125.7,125.5,124.9,122.0,121.9,121.0,121.0,112.0,83.0$, 61.0, 13.7.

HRMS (MALDI) Calcd. for $\mathrm{C}_{62} \mathrm{H}_{46} \mathrm{O}_{6}: 909.3187$ (MNa ${ }^{+}$). Found: 909.3177 (100\%).

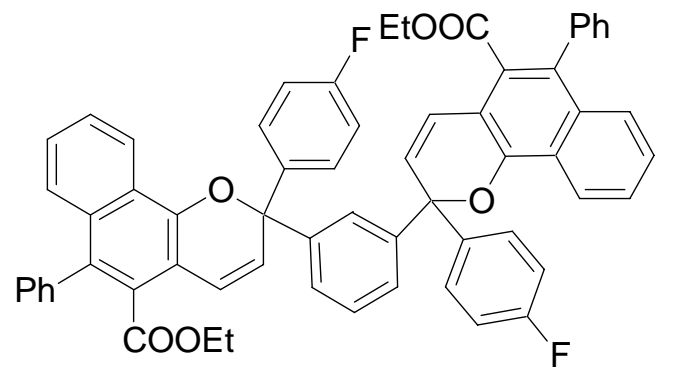

3'b, 1,3-di-(2-p-fluorophenyl-6-phenyl-5-ethoxycarbonyl-[2H]-naphtho[1,2-b]pyran-2yl)-benzene

The general procedure was followed using 1,3- di[1-hydroxy-1-( $p$-fluorophenyl)-prop-2ynyl]benzene and 4-Hydroxy-1-phenyl-naphthoic acid ethyl ester (3 equiv) as starting material and the mixture was refluxed for $6 \mathrm{~h}$, pale orange foam was obtained in yield of $72 \%$.

IR (KBr): 3059, 2927, 1721, 1601, 1506, 1445, 1430, 1393, 1371, 1292, 1227, 1158, 1113, 1041, 1002, 836, 771, 702.

${ }^{1} \mathrm{H}$ NMR $\left(300 \mathrm{MHz}, \mathrm{CDCl}_{3}\right): \delta 8.28-8.18(\mathrm{~m}, 4 \mathrm{H}), 7.76-7.63(\mathrm{~m}, 1 \mathrm{H}), 7.55-7.28(\mathrm{~m}$, 24H), 6.96-6.86 (m, 4H), $6.81(\mathrm{~d}, \mathrm{~J}=10.0 \mathrm{~Hz}, 1 \mathrm{H}), 6.80(\mathrm{~d}, \mathrm{~J}=10.0 \mathrm{H} \mathrm{z}, 1 \mathrm{H}), 6.13(\mathrm{~d}, \mathrm{~J}=$ $10.0 \mathrm{~Hz}, 1 \mathrm{H}), 6.12(\mathrm{~d}, \mathrm{~J}=10.0 \mathrm{~Hz}, 1 \mathrm{H}), 4.01(\mathrm{q}, \mathrm{J}=7.2 \mathrm{~Hz}, 4 \mathrm{H}), 0.89(\mathrm{t}, \mathrm{J}=7.2 \mathrm{~Hz}, 6 \mathrm{H})$.

${ }^{13} \mathrm{C}$ NMR $\left(75 \mathrm{MHz}, \mathrm{CDCl}_{3}\right): \delta 168.1,161.9\left({ }^{1} \mathrm{~J}=246.6 \mathrm{~Hz}\right), 147.0,144.5,144.5,140.30$ $\left({ }^{4} \mathrm{~J}=3.0 \mathrm{~Hz}\right), 140.22\left({ }^{4} \mathrm{~J}=3.0 \mathrm{z}\right), 138.0,132.7,132.7,131.1,130.4,128.6\left({ }^{3} \mathrm{~J}=8.5 \mathrm{~Hz}\right)$, 128.2 , 128.1, 127.9, 127.5, 127.5, 127.3, 127.0, 126.9, 126.9, 126.8, 126.6, 126.2, 126.1, 125.6, 125.2, 124.8, 121.7, 121.7, 121.3, 121.3, $115.0\left({ }^{2} \mathrm{~J}=21.4 \mathrm{~Hz}\right), 112.1,112.0,82.6$, $61.1,13.7$. 
HRMS (MALDI) Calcd. for $\mathrm{C}_{62} \mathrm{H}_{44} \mathrm{~F}_{2} \mathrm{O}_{6}: 945.2998\left(\mathrm{MNa}^{+}\right.$). Found: 945.3009 (100\%).

\section{Spectroscopic characteristics of bispyran}

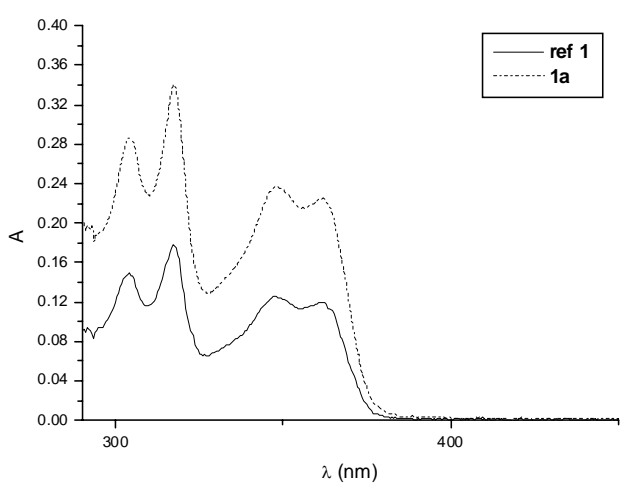

Figure 1. UV-vis spectra of compound $\mathbf{1 a}$ and $\mathbf{6}$ (both $2.5 \times 10^{-5} \mathrm{~mol} / \mathrm{L}$ in toluene).

The absorption of 1a has four absorption bands in the region 300-400 nm: $304 \mathrm{~nm}$ (11400), $317 \mathrm{~nm}$ (13600), $347 \mathrm{~nm}$ (9500), and $361 \mathrm{~nm}(9000)$, 1a has same absorption maxima as reference compound 3,3-diphenyl-[3H]-naphtho[2,1-b]pyran (6) $304 \mathrm{~nm}$ (6000), $317 \mathrm{~nm}$ (7400), $347 \mathrm{~nm}$ (5200), and $361 \mathrm{~nm}$ (4900), however with much higher extinction coefficient. This indicates that the two photochromic units in 1a are well insulated, and no communication between the two pyran units in the closed forms.

\section{General procedure for low-temperature chromatography}

A solution of photochromic compound in a mixture of hexane/ $\mathrm{CH}_{2} \mathrm{Cl}_{2}(1: 1)$ in a quartz container irradiated with UV (366 nm) was loaded onto a silica gel column fitted with a cooling jacket which was filled with dry ice-acetone under slight vacuum, then the column was eluted cold dichloromethane under slight vacuum. The collected fractions in thick-wall tubes were immediately frozen with liquid nitrogen. The frozen fraction isolated by low-temperature chromatography was transferred into a cuvette cooled in Oxford Cryostat while the frozen solution partially melted, then UV-vis spectra were recorded in Cary 50 with Oxford Cryostat at $-78{ }^{\circ} \mathrm{C}$. The cuvette containing the colored solution was warmed to room temperature in the dark till all the color completely faded (the residue color may also be bleached with visible light). The faded solution was then measured with UV-vis spectrometer.

\section{(a) Separation of closed-TC.}

The solution of compound $\mathbf{1 b} / \mathbf{1} \mathbf{b}$ ' in hexane/dichloromethane irradiated with UV light for $5 \mathrm{sec}$ at temperature $\leq-30{ }^{\circ} \mathrm{C}$ was loaded on the silica gel column, and the colored form was separated following the general procedure. The spectra of the isolated isomer of closed-TC for $\mathbf{1} \mathbf{b}$ and $\mathbf{1} \mathbf{b}$ are shown in Figure 2. There is little difference of $\lambda_{\max }$ between the closed-TC forms of $\mathbf{1 b}(445 \mathrm{~nm})$ and $\mathbf{1}^{\prime} \mathbf{b}(443 \mathrm{~nm})$. 

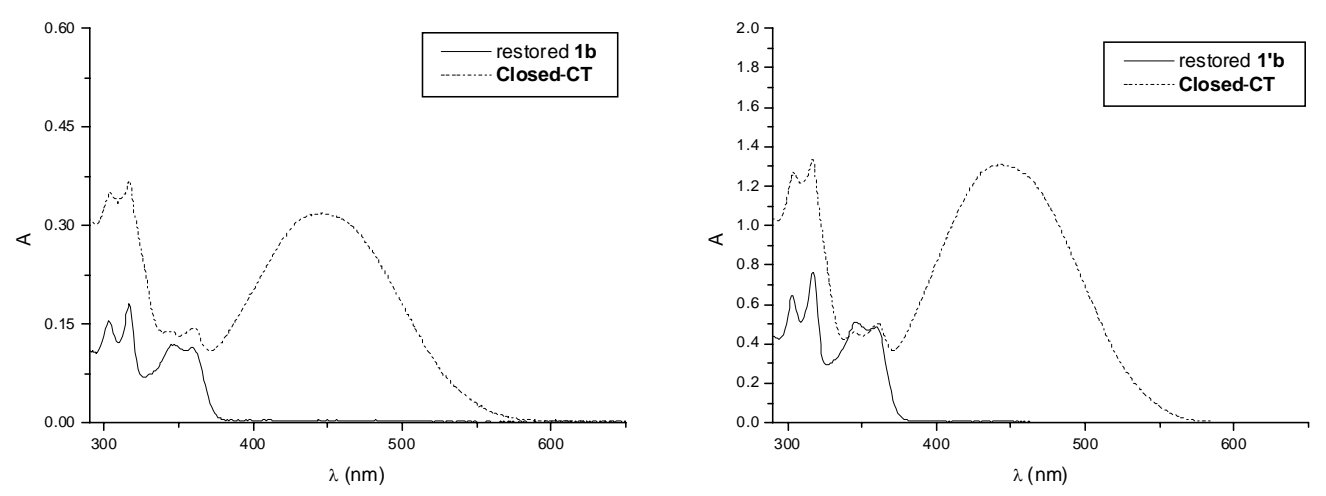

Figure 2. Absorption spectra of the colored species of $\mathbf{1 b}$ and $\mathbf{1}$ 'b isolated by lowtemperature chromatography on silica gel at $-78{ }^{\circ} \mathrm{C}$ in dichloromethane, and the regenerated bispyrans at ambient temperature.

\section{(b) Separation of closed-TT.}

The solution of compound $\mathbf{1 b} / \mathbf{1} \mathbf{b}$ ' in hexane/dichloromethane was irradiated with UV light for $50 \mathrm{sec}$ at $20^{\circ} \mathrm{C}$, the fading of color was monitored with Cary 50 at $510 \mathrm{~nm}$ till very slow fading was observed. The partially faded solution was resubmitted to another cycle of irradiation along with fading as described above, and then the colored solution was loaded on a pre-cooled column $\left(-78^{\circ} \mathrm{C}\right)$ of silica gel, and was separated following the general procedure. Little difference of $\lambda_{\max }$ between the closed-TT forms of $\mathbf{1 b}$ (440 $\mathrm{nm}$ ) and $\mathbf{1}$ 'b (435 nm) was observed.
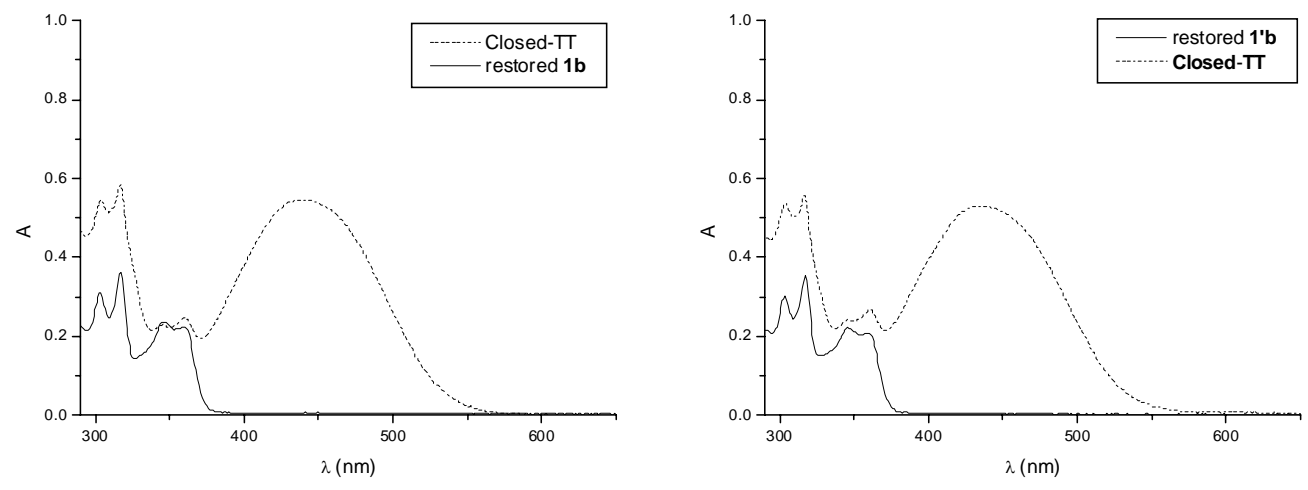

Figure 3. Absorption spectra of the colored species of $\mathbf{1 b}$ and $\mathbf{1}$ 'b isolated by lowtemperature chromatography on silica gel at $-78^{\circ} \mathrm{C}$ in dichloromethane, and the restored bispyrans at ambient temperature.

\section{Calculation of extinction coefficient:}

The spectra of isolated colored forms of $\mathbf{1 b}$ or $\mathbf{1 b}$ ' have the same shape as colored forms generated at room temperature by UV, however with slightly shifted absorption maxima (less than $12 \mathrm{~nm}$ ). The absorbance was recorded at $-78{ }^{\circ} \mathrm{C}$ (the spectra taken at room temperature are $85 \%$ intensity of those taken at $-78^{\circ} \mathrm{C}$ due to the change of the volume of the solvent). The colored forms isolated were then warmed up to room temperature, and 
was kept in the dark till complete fading of the color (if some residue color or closed-TT remains, it could be bleached by irradiation with visible light). The restored absorption spectrum of bisnaphthopyran 1b or 1b' was compared with that of the standard solution of $1 \mathrm{~b}$ or $1 \mathrm{~b}$ ', so that the concentration the restored $1 \mathrm{~b}$ or $1 \mathrm{~b}^{\prime}$ could be obtained which corresponding to the concentration of the colored form isolated. Thus the extinction coefficient could be calculated to be 21300 and $20000 \mathrm{dm}^{3} \cdot \mathrm{mol}^{-1} \cdot \mathrm{cm}^{-1}$ respectively for the colored forms (closed-TC) of $\mathbf{1 b}$ and 1b', and 18300 and 16800 for the closed-TT forms of $\mathbf{1 b}$ and $\mathbf{1}$ 'b, respectively.

\section{Photocoloration and quantum yield measurements:}

The absorption maxima of the colored species generated at room temperature are slightly different from those isolated at $-78{ }^{\circ} \mathrm{C}(<12 \mathrm{~nm}$ shift for the long absorption band). We assume that such a small difference of absorption maxima will not affect the value of $\varepsilon$. At early stage of the photocoloration reaction, mostly closed-TC is generated similar to the discovery in pyran, ${ }^{3}$ thus the photochromic reaction is simplified as:

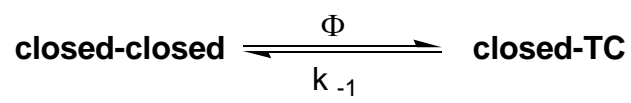

From eq (1), the following equation could be obtained: ${ }^{4}$

$\mathrm{dA}_{\text {closed-TC }} / \mathrm{dt}=\varepsilon_{\text {closed-TC }} \Phi \mathrm{I}_{0}\left[1-\exp \left(-2.3 \mathrm{~A}_{\text {closed-closed }}\right)\right]-\mathrm{k}_{-1} \mathrm{~A}_{\text {closed-TC }}$

Wherein $\mathrm{A}_{\text {closed-TC }}$ is the absorbance of closed-TC at absorption maximum. $\varepsilon_{\text {closed-TC }}$ is the extinction coefficient of closed-TC; $\Phi$ is the quantum yield of the photo reaction. $\mathrm{I}_{0}$ is the intensity of the light. $\mathrm{A}_{\text {closed-closed }}$ is the absorbance of closed-closed at the irradiation wavelength $(366 \mathrm{~nm})$. By plotting $\mathrm{dA}_{\text {closed-TC }} / \mathrm{dt}$ versus $\mathrm{A}_{\text {closed-closed }}$ and extrapolating the color-forming rate to zero time, the quantum efficiency $\Phi$ could be calculated by the following equation.

$\Phi=\left(\mathrm{dA}_{\text {closed-TC }} / \mathrm{dt}\right)_{\mathrm{t} \rightarrow 0} /\left\{\varepsilon_{\text {closed-TC }} \mathrm{I}_{0}\left[1-\exp \left(-2.3 \mathrm{~A}_{\text {closed-closed }}\right)\right]\right\}$

Photocoloration kinetic measurements were carried out a thermostated cuvette irradiated with UV (366 nm) from $150 \mathrm{~W}$ medium pressure $\mathrm{Hg}$ lamp filtered through the combination of y $5.0 \times 10^{-5} \mathrm{~mol} / \mathrm{L}$ solution of photochromic compound in dichloromethane. Irradiation beam of UV is at $90^{\circ}$ angle to the monitoring beam and the absorbance was monitored at the absorption maximum of the colored form with a Cary $50 \mathrm{UV}$-vis spectrometer. The light intensity was measured with potassium ferrioxalate actinometer following standard procedure to be $7.98 \times 10^{-6}$ einstein $\cdot \mathrm{s}^{-1} \cdot \mathrm{dm}^{-3}$. 

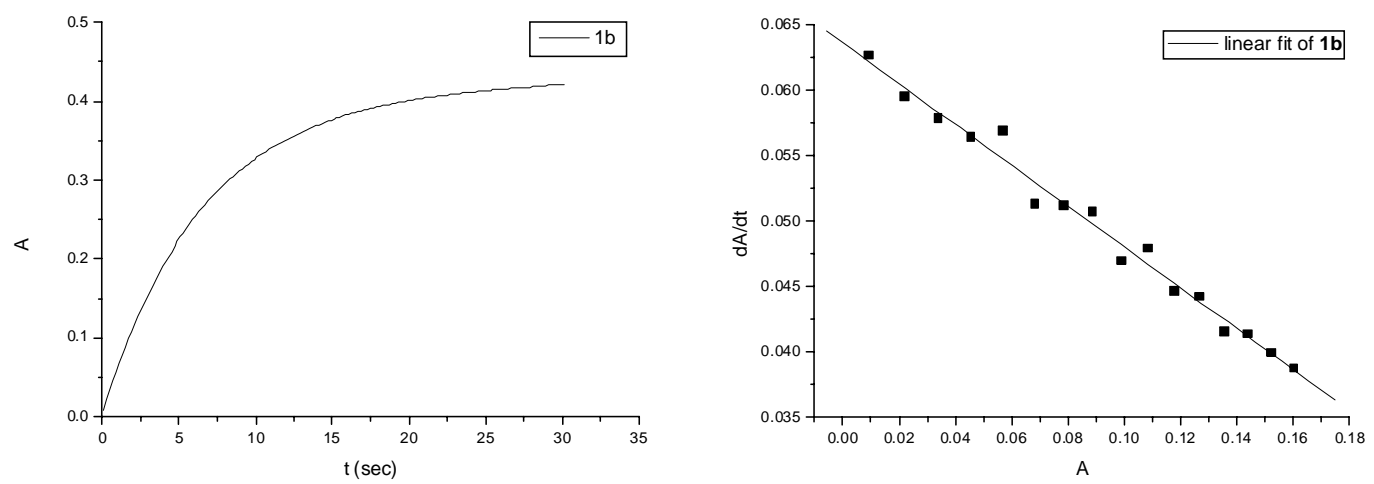

Figure 4. Photocoloration of compound $\mathbf{1 b}$ in dichloromethane $\left(5 \times 10^{-5} \mathrm{~mol} / \mathrm{L}\right)$ at $300 \mathrm{~K}$. (a) Absorbance of the colored forms at $433 \mathrm{~nm}$ upon UV irradiation; (b) Experimental data treated according eq (2).
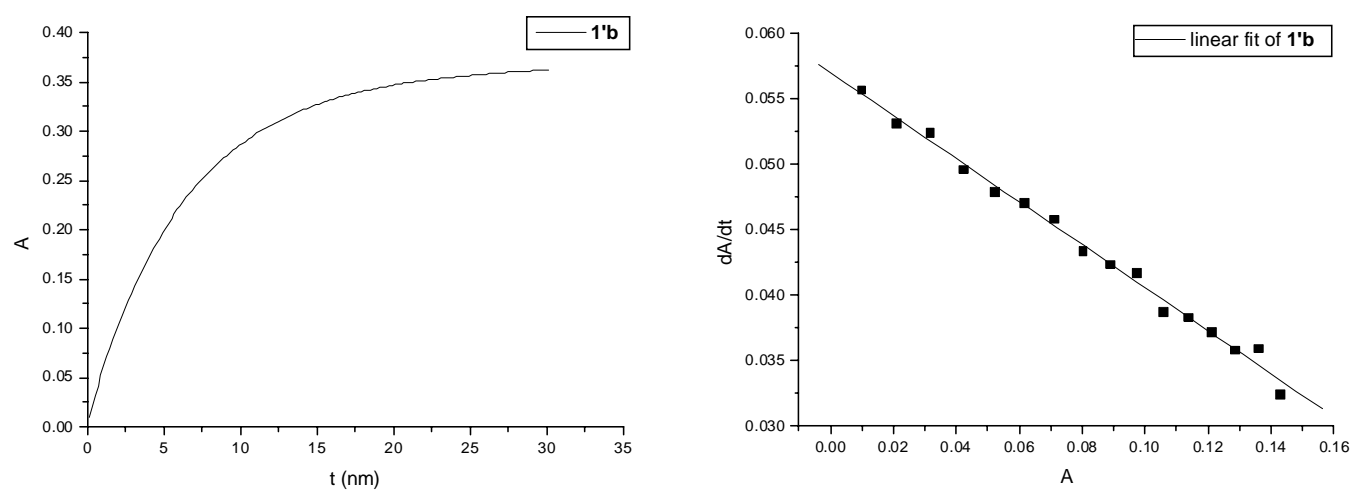

Figure 5. Photocoloration of compound $\mathbf{1 b}$ ' in dichloromethane $\left(5 \times 10^{-5} \mathrm{~mol} / \mathrm{L}\right)$ at 300 K. (a) Absorbance of the colored forms at $430 \mathrm{~nm}$ upon UV irradiation; (b) Experimental data treated according eq (2).

$\left(\mathrm{dA}_{\text {closed-TC }} / \mathrm{dt}\right)_{\mathrm{t} \rightarrow 0}$ could be obtained from linear fitting of photocoloration shown in Figure 4 (b). When the change of absorbance (dA/dt) was plotted against absorbance (A), very good linear relationship with correlation coefficient better than 0.99 was obtained, and thus $\left(\mathrm{dA}_{\text {closed-TC }} / \mathrm{dt}\right)_{\mathrm{t} \rightarrow 0}$ was calculated to be 0.063 . Two parallel measurements were performed and the calculated value of $\Phi$ is $0.720 \pm 0.003$ for $\mathbf{1 b}$. Similarly, from Figure 5, $\quad\left(\mathrm{dA}_{\text {closed-TC }} / \mathrm{dt}\right)_{\mathrm{t} \rightarrow 0}$ was calculated to be 0.057 , and $\Phi$ for $\mathbf{1} \mathbf{b}$ is $0.723 \pm 0.005$.

\section{Thermofading kinetic measurements:}

$5.0 \times 10^{-5} \mathrm{~mol} / \mathrm{L}$ solution of photochromic compound in dichloromethane in thermostated cuvette was irradiated for $5 \mathrm{sec}$ with UV $(366 \mathrm{~nm})$. Once the light source was removed, the absorbance was monitored with time by Cary 50 at the absorption maximum of the colored form. The temperature was controlled with thermo-cryostat.

In general biexponential fading processes are involved. Upon short period time of irradiation ( $\mathrm{t} \leq 6 \mathrm{sec}$ ), due to the low content of slow fading closed-TT, the fading process is dominated by closed-TC and could be simplified as monoexponential process. 
The fading of color monitored at $434 \mathrm{~nm}$ at $298.4 \mathrm{~K}$ for a $50 \mu \mathrm{M}$ solution of compound 1b in dichloromethane after $5 \mathrm{sec}$ of UV $(366 \mathrm{~nm})$ irradiation is shown in Figure 6 (a). Mathematic simulation afforded the thermal fading for closed-TC of $\mathbf{1 b}$ to be $0.135 \mathrm{~s}^{-1}$ at 298.4K. Similarly, the fading rate for closed-TC of $\mathbf{1}$ 'b was obtained to be $0.158 \mathrm{~s}^{-1}$ at 298.4K.
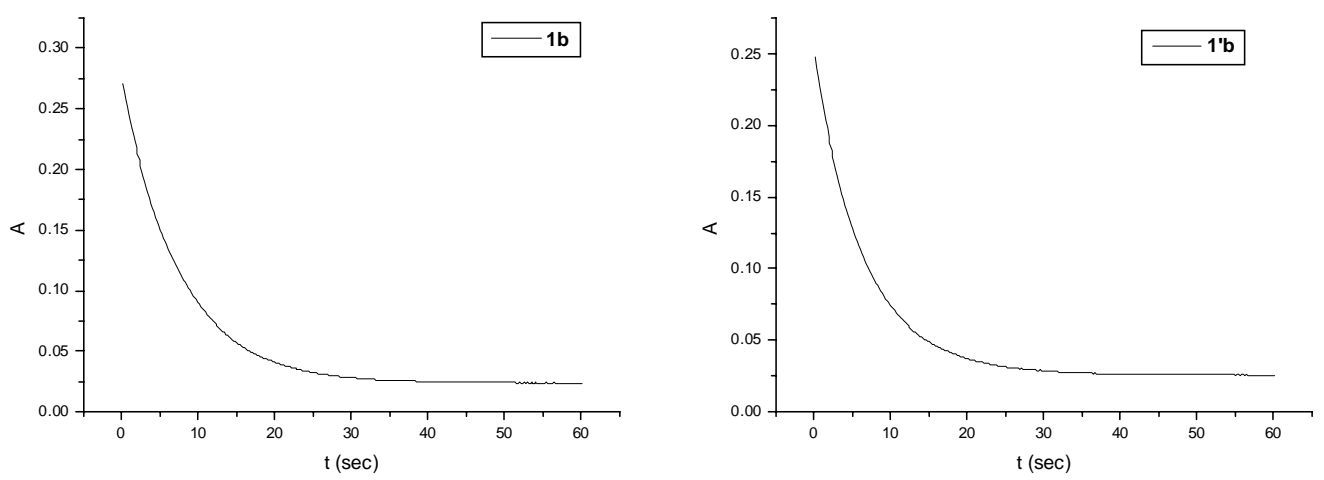

Figure 6. Thermal fading of compound $\mathbf{1 b}$ and $\mathbf{1 b}$ ' in dichloromethane $\left(5 \times 10^{-5} \mathrm{~mol} / \mathrm{L}\right)$ at 298.4 K monitored at $434 \mathrm{~nm}$ after UV irradiation for $5 \mathrm{sec}$.

\section{Fatigue-resistance measurements:}

A solution of photochromic compound in toluene was equilibrated in a thermostated cuvette for $0.5 \mathrm{~h}$ prior to photoirradiation. It was irradiated for 4 min under magnetic stirring with white light from $150 \mathrm{~W}$ Xenon lamp which was passed through a water circulated heat trap and $\mathrm{C} 3 \mathrm{C} 26$ optical filter to eliminate heat, infrared light, and UV light less than $300 \mathrm{~nm}$, then the absorption was started to be monitored with Cary 50 at the $\lambda$ max of the colored forms. The output of the white light was measured with EPM1000 power meter. The normalized curves for the degradation of $\mathbf{1 b}$ and $\mathbf{1 b}$ ' as well as 3,3-diphenyl-[3H]naphtha[2,1-b]pyran (6) are shown in Figure 7. Dramatic difference between $\mathbf{1 b}$ and $\mathbf{1 b}$ ' was observed. 


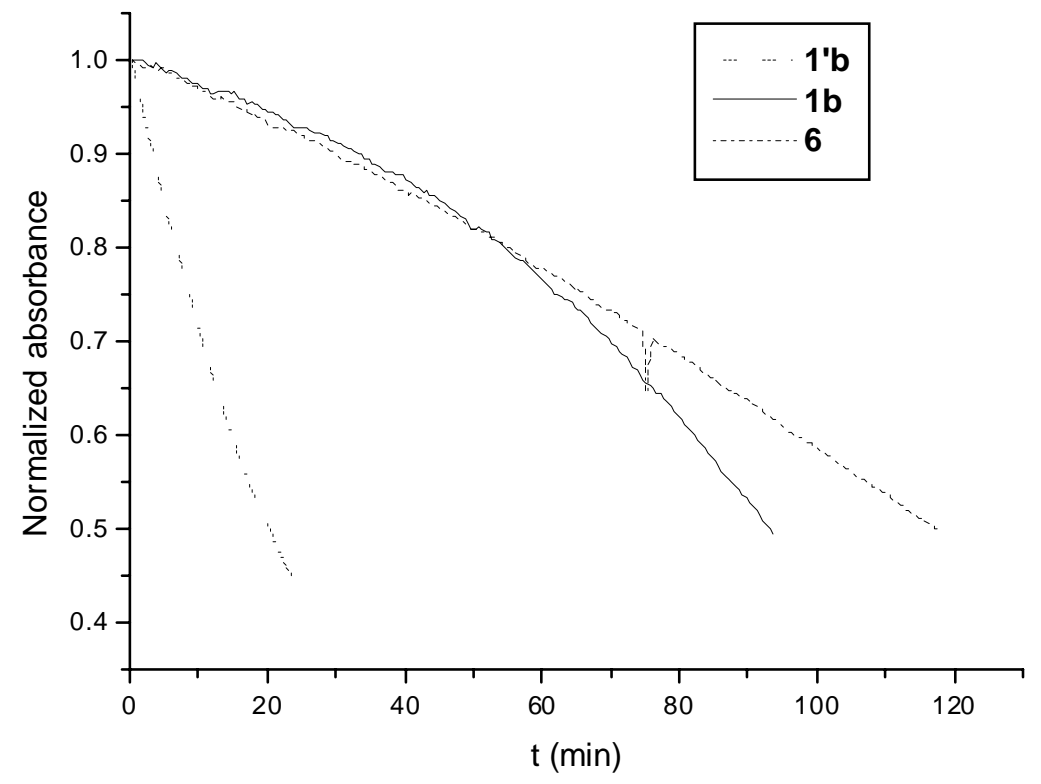

Figure 7. Degradation of the colored forms of compound $\mathbf{1 b}$ and $\mathbf{1 b}$ ' as well as reference compound 3,3-diphenyl-[3H]naphtha[2,1-b]pyran (6) in toluene $\left(2.5 \times 10^{-5} \mathrm{~mol} / \mathrm{L}\right)$ under white light irradiation at $298.4 \mathrm{~K}$ monitored at $434 \mathrm{~nm}$.

\section{Reference:}

(1) Heller, H.G.; Levell, J. R. U.S. Patent 5,955,520, 1999.

(2) Zhao, W.; Carreira, E. M. Org. Lett. 2003, 5, 4153-4154.

(3) (a) Frioli, M.; Pimienta, V.; Moustrou, C.; Samat, A.; Guglielmetti, R.; Aubard, J.; Maurel, F.; Micheau, J. C. Photochem. Photobiol. Sci. 2003, 2, 888-892. (b) Delbaere, S.; Micheau, J. C.; Vermeersch, G. J. Org. Chem. 2003, 68, 8968-8973.

(4) (a) Ottavi, G.; Ortica, F.; Favaro, G. Int. J. Chem. Kinet. 1999, 31, 303-313. (b) Becker, R. S.; Pelliccioli, A. P.; Romani, A.; Favaro, G. J. Am. Chem. Soc. 1999, 121, 2104-2109.

(5) Murov, S. L.; Carmichael, L.; Hug, G. L. Handbook of Photochemistry, Marcel Dekker, Inc. 1993. pp 298-305. 